Supplement of

\title{
Characterization and origin of the Mn-rich patinas formed on Lunéville château sandstones
}

\section{Laure Gatuingt et al.}

Correspondence to: Laure Gatuingt (laure.gatuingt@gmail.com) and Bruno Lanson (bruno.lanson@univ-grenoble-alpes.fr)

The copyright of individual parts of the supplement might differ from the article licence. 
Area not affected by the 2003 fire

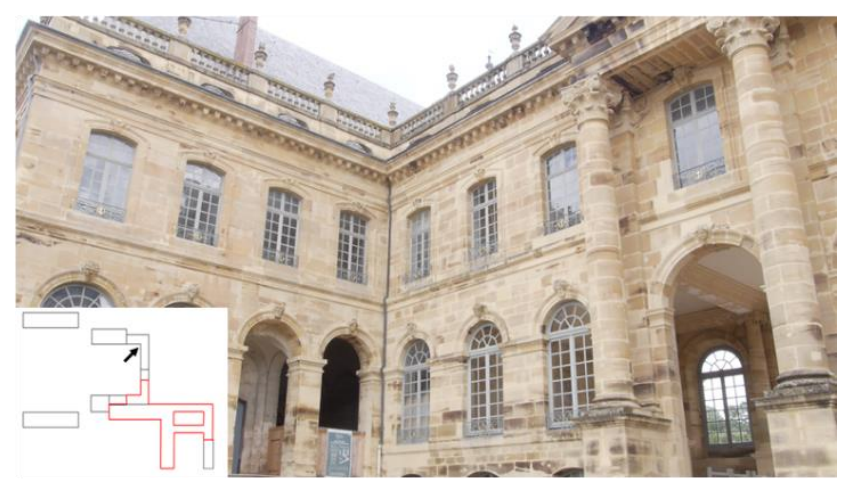

Area affected by the 2003 fire
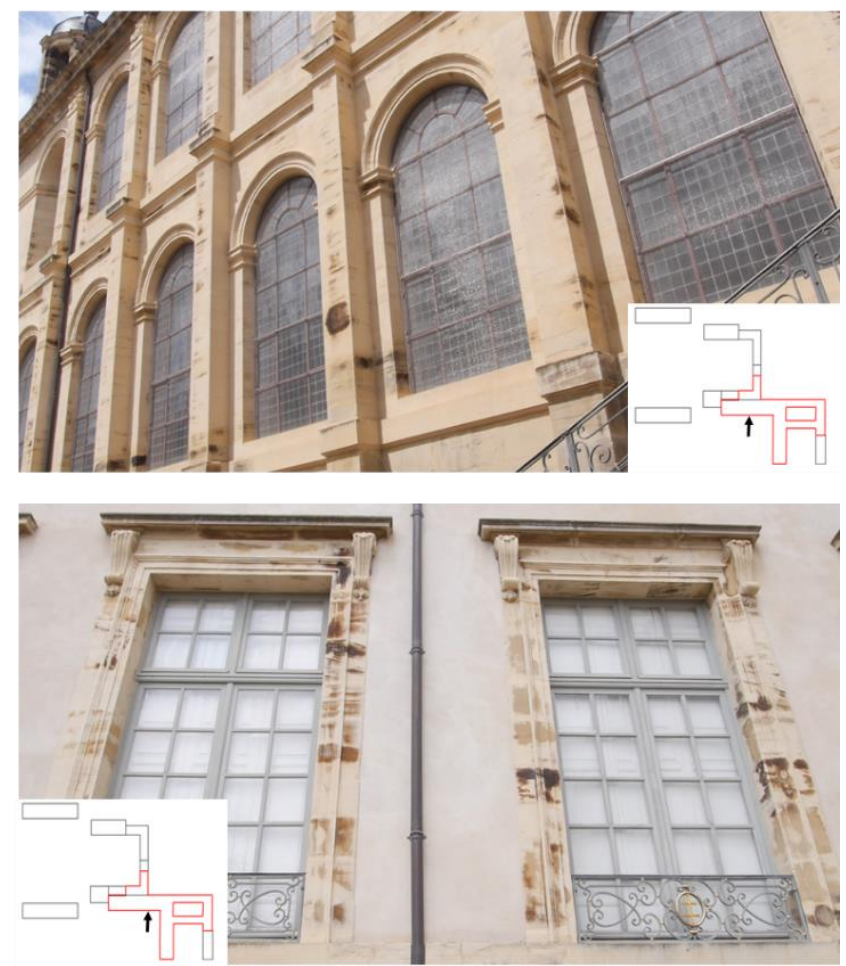

25 Figure SI1: Photographs of the Lunéville château and their location on the sketch of the château (the zone in red represents the zone affected by the 2003 fire). Dark patinas affect only specific sandstones blocks. 
L1 bulk-ROI 1

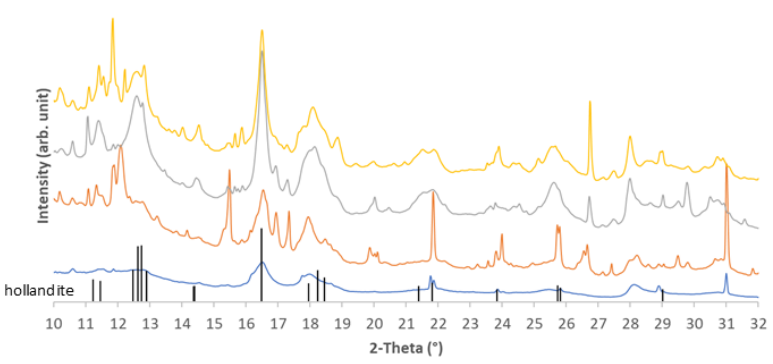

L1 bulk-ROI 2

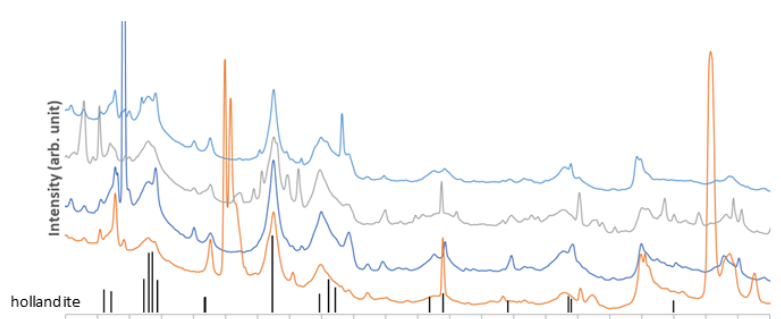

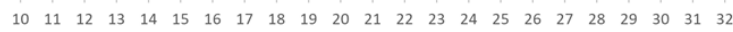
2-Theta $\left({ }^{\circ}\right)$
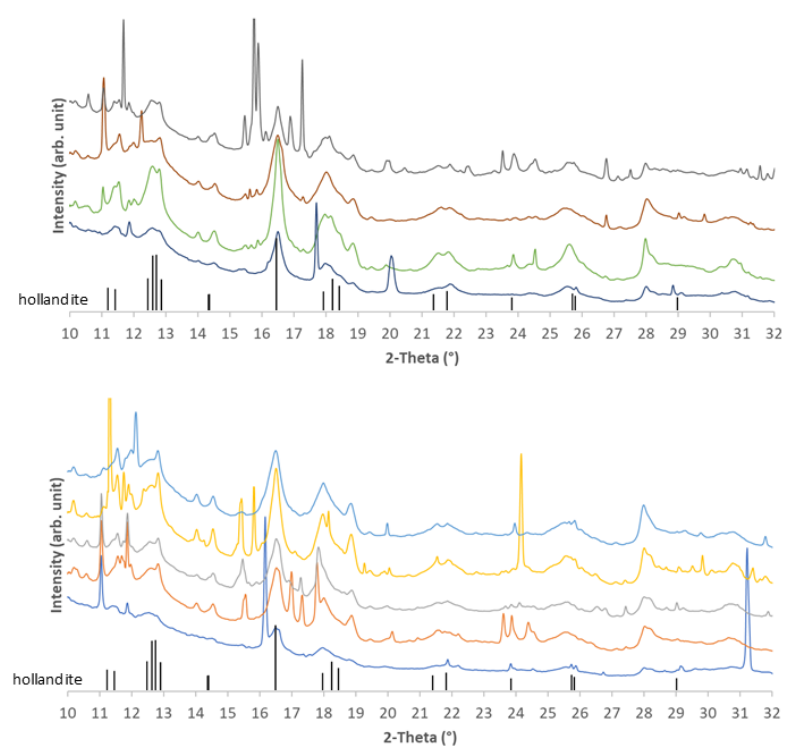

30 Figure SI2: X-ray diffraction patterns obtained in Mn-rich regions of interest (ROIs) of L1 bulk. A total of 17 usable XRD patterns were collected in two ROIs: 4 in ROI 1 and 13 in ROI 2. "ROI n" refers to black Mn-rich areas similar to those shown in figure 5 . 
L1 deep bulk

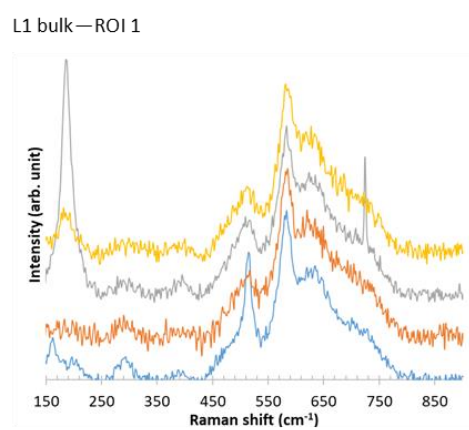

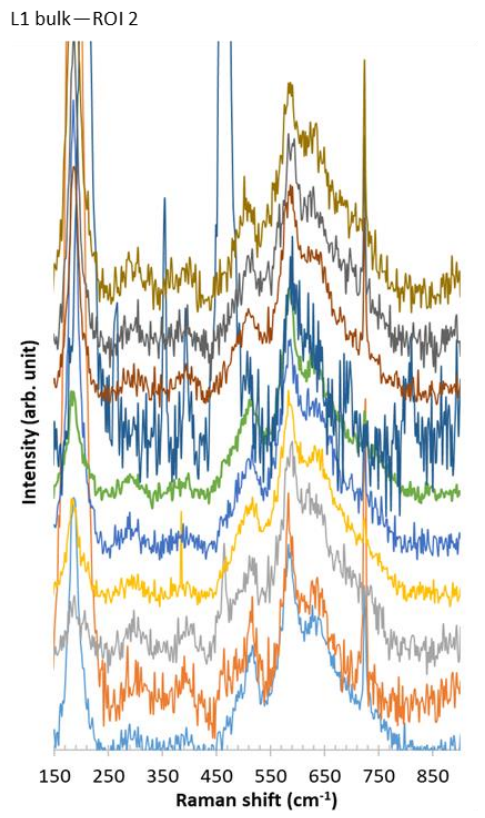

L1 bulk

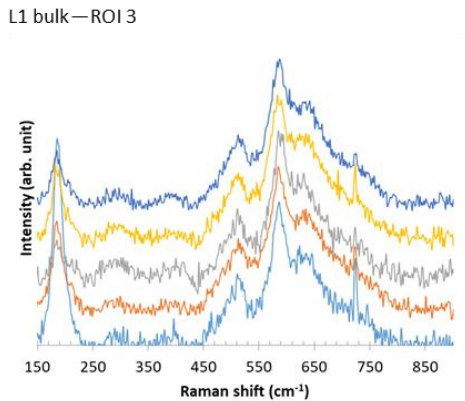

L1 subsurface

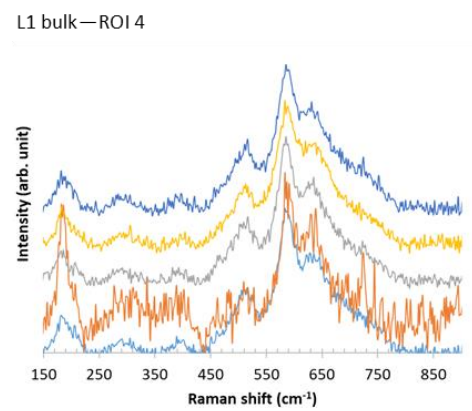

Figure SI3: Raman spectra obtained in Mn-rich regions of interest (ROIs) of L1 bulk. 24 usable Raman spectra were collected in four ROIs: 4 in ROI 1, 10 in ROI 2, 5 in ROI 3 and 5 in ROI 4. Comparison of a representative Raman spectrum obtained from L1 bulk with that of natural hollandite is illustrated in figure 7c. "ROI n" refers to black Mnrich areas similar to those shown in figure 5. 

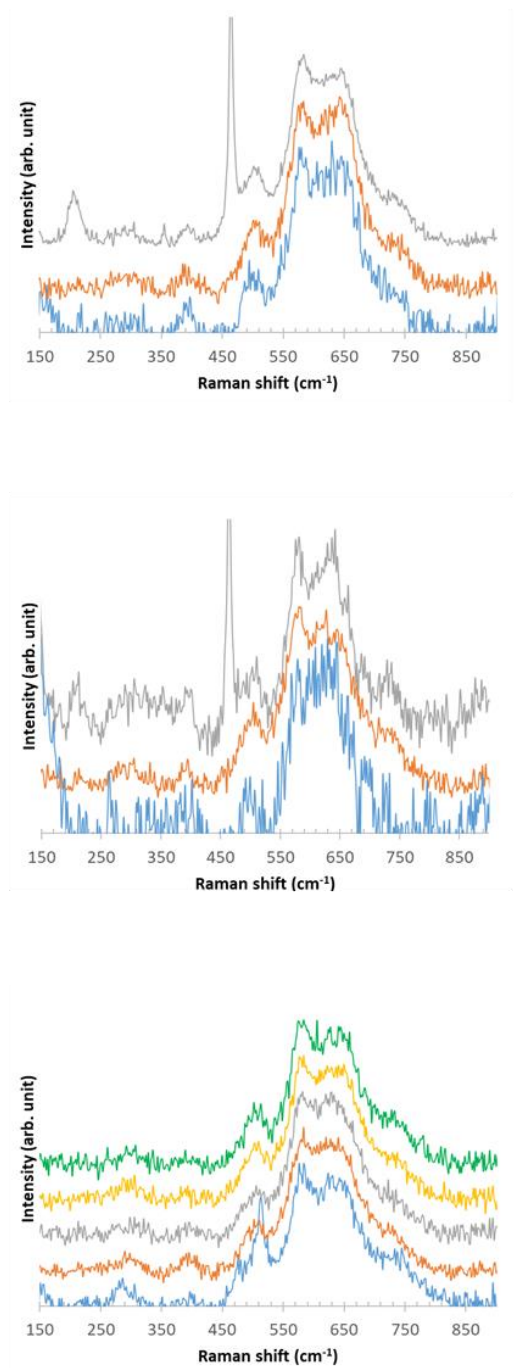

Figure SI4: Raman spectra obtained on L1 patina. 


\section{L1 patina}
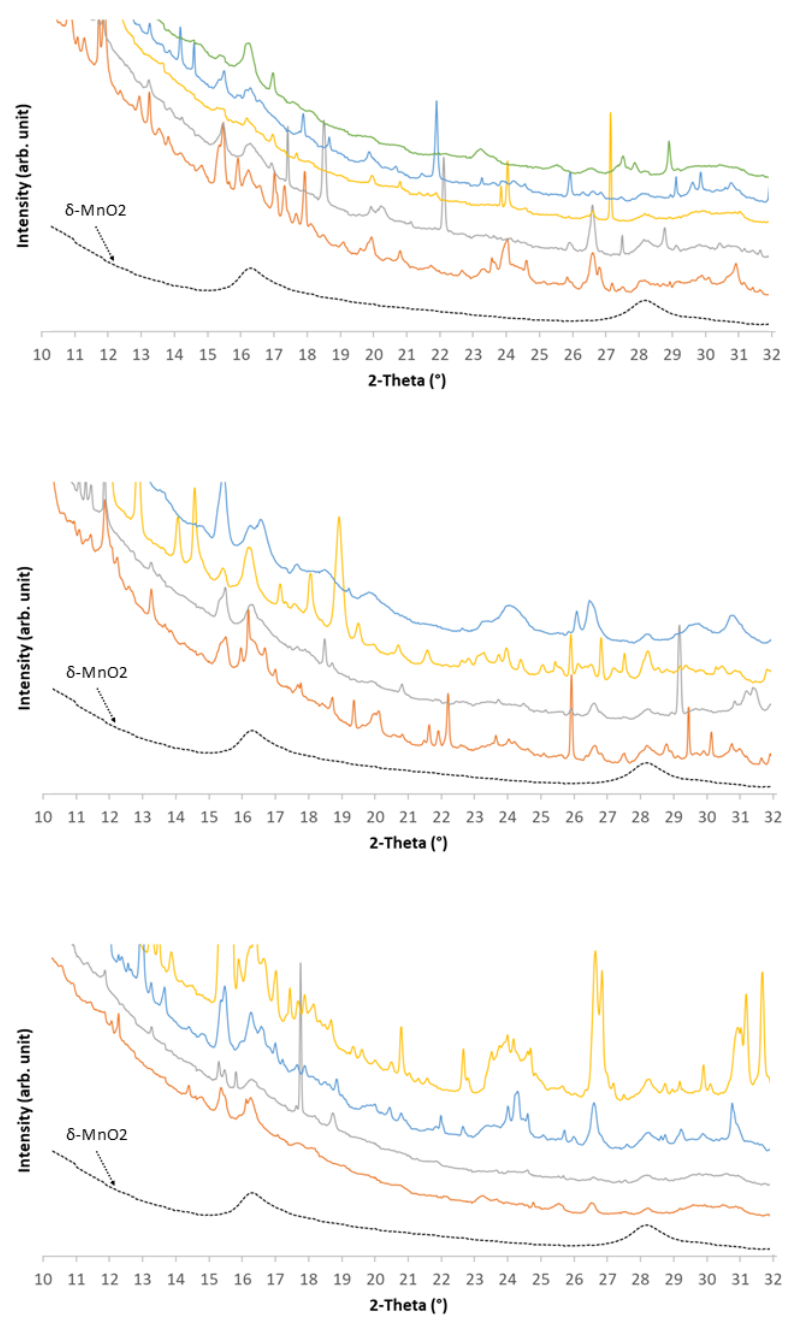

Figure SI5: X-ray diffraction patterns obtained on L1 patina. 